\title{
UTILIZAÇÃO DO DIAGNÓSTICO DE ENFERMAGEM SEGUNDO A CLASSIFICAÇÃO DA NANDA, PARA A SISTEMATIZAÇÃO DA ASSISTÊNCIA DE ENFERMAGEM EM ALEITAMENTO MATERNO *
}

\author{
Ana Cristina F. Vilhena Abrão ** \\ Maria Gaby R. de Gutiérrez **** \\ Heimar de Fátima Marin ****
}

ABRÃO, A.C.F.V.; GUTIÉRREZ, M.G.R.de.; MARIN, H.F. Utilização do diagnóstico de enfermagem segundo a classificação da NANDA, para a sistematização da assistência de enfermagem em aleitamento materno. Rev.latinoam.enfermagem, Ribeirão Preto, v. 5, n. 2, p. 49-59, abril 1997.

Este estudo teve por objetivos descrever a reformulação de um instrumento utilizado na consulta de enfermagem à puérpera, com base na classificação dos diagnósticos de enfermagem, segundo a Taxonomia I-revisada da American Nursing Diagnosis Association - NANDA, e identificar os diagnósticos de enfermagem mais frequentes, relacionados ao aleitamento materno a partir da utilização deste instrumento. Os diagnósticos encontrados em percentuais acima de 50\% foram: déficit de conhecimento (100,0\%); distúrbio no padrão do sono (75,0\%); alteração no padrão de sexualidade (75,0\%); amamentação ineficaz $(66,6 \%)$ e mobilidade física prejudicada $(66,6 \%)$.

UNITERMOS: diagnóstico de enfermagem, aleitamento materno, saúde da mulher

\section{INTRODUÇÃO}

Neste estudo descritivo, são abordados alguns aspectos teóricos e práticos relacionados à identificação de diagnósticos de enfermagem em aleitamento materno.

$\mathrm{O}$ aleitamento materno deve ser visto como prática indispensável para a melhoria da saúde e qualidade de vida das mães e crianças. As estratégias de promoção desta prática que vêm sendo utilizadas há, aproximadamente, duas décadas enfatizam a necessidade de conscientizar a população sobre as inúmeras vantagens oferecidas pelo leite materno, em relação a outros tipos de leite (GIUGLIANI $\left.{ }^{7}, 1994\right)$.

Concordamos com CAETANO ${ }^{2}$ (1992) que ressalta que a prática ao aleitamento materno é bastante complexa pois deve contemplar não apenas os aspectos biológicos, mas também os psicológicos e sócio-culturais nele envolvidos. $\mathrm{O}$ ato de amamentar não está relacionado apenas as questões biológicas, mas sobretudo a um conjunto de fatores que contribuem ou interferem nesta prática para o seu êxito.
A formação de recursos humanos para o incentivo ao aleitamento materno e assistência à mulher que deseja amamentar ou que está amamentando, é uma das estratégias propostas pela Organização Mundial da Saúde (OMS/UNICEF ${ }^{12}$, 1989).

O enfermeiro como profissional de saúde, tem a oportunidade de realizar não somente ações educativas, mas sobretudo assistenciais, especificamente na prevenção e tratamento dos traumas mamilares, ingurgitamento mamário e mastite, patologias comuns no início da amamentação, responsáveis, algumas vezes, pelo desmame precoce (MANFREDI ${ }^{11}$, 1989).

Desta forma, as ações de enfermagem relacionadas ao aleitamento materno devem ser realizadas de forma sistematizada. Acredita-se que a utilização do diagnóstico como etapa do processo de enfermagem, no atendimento ao binômio mãe-filho, durante o período de internação ou nas consultas de enfermagem de retorno ambulatorial, possa contribuir para uma assistência mais direcionada e eficaz.

* Trabalho apresentado na Disciplina "Estudos Avançados da Assistência de Enfermagem”, no Curso de Doutorado em Enfermagem do Departamento de Enfermagem da Universidade Federal de São Paulo

** Professor Assistente da Disciplina de Enfermagem Obstétrica do Departamento de Enfermagem da Universidade Federal de São Paulo

*** Professor Adjunto da Disciplina Fundamentos de Enfermagem e Enfermagem Médico Cirúrgica do Departamento de Enfermagem da Universidade Federal de São Paulo

**** Professor Adjunto da Disciplina de Enfermagem Obstétrica do Departamento de Enfermagem da Universidade Federal de São Paulo 
O termo diagnóstico surgiu na literatura norteamericana em 1950, quando Mac Manus propôs, dentre as responsabilidades do enfermeiro, a identificação dos diagnósticos ou problemas de enfermagem ${ }^{* * * * * *}$. Mais recentemente, a partir da década de 70 , estudos vêm sendo desenvolvidos com o objetivo de estabelecer uma Classificação Internacional dos Diagnósticos de Enfermagem (FARIAS ${ }^{5}$ et al., 1990; CRUZ ${ }^{4,3}, 1994$, 1995).

A partir de 1973, várias conferências foram realizadas e muitos avanços foram sendo conseguidos no sentido de definir melhor quais os diagnósticos a serem identificados e como validá-los na prática clínica (FARIAS $^{5}$ et al., 1990; CRUZ $^{3}$, 1995).

A Associação Norte-Americana de Diagnóstico de Enfermagem (NANDA), criada em 1982, assumiu a responsabilidade de dar continuidade aos trabalhos até então desenvolvidos neste sentido (FARIAS ${ }^{5}$ et al., 1990; IYER $^{9}$ et al., 1993; CRUZ $\left.{ }^{4,3}, 1994,1995\right)$.

Em decorrência destes trabalhos, foi estabelecida em 1986 a primeira Classificação Internacional denominada Taxonomia I dos diagnósticos de Enfermagem, substituída pela Taxonomia I - revisada em 1989, com inclusão de algumas alterações e, atualmente já foi definida a taxonomia II (FARIAS ${ }^{5}$ et al., 1990; $\left.\mathrm{CRUZ}^{3}, 1995\right)$.

No Brasil, a expressão diagnóstico de enfermagem foi introduzida por Wanda Horta, na década de 60, e constituiu-se em uma das etapas do processo de enfermagem proposto por esta autora (HORTA $\left.{ }^{8}, 1979\right)$.

Atualmente, este tema tem sido objeto de discussão em vários eventos, visando melhor compreensão e aplicação da Taxonomia dos diagnósticos de enfermagem propostos pela NANDA (CRUZ ${ }^{4,3}, 1994$, 1995).

No que diz respeito a utilização dos diagnósticos de enfermagem em aleitamento materno, segundo a classificação da NANDA, dentre as publicações existentes, podemos citar o trabalho de validação do diagnóstico "amamentação ineficaz" realizado por LETHBRIGE $^{10}$ et al., (1993). Inúmeros outros trabalhos de validação estão sendo realizados no sentido de se obter uma confirmação, na prática, da classificação existente.

A experiência de uma das autoras do presente trabalho, na assistência sistematizada em aleitamento materno, fundamentada no modelo proposto por HORTA ${ }^{8}$ (1979), permitiu concluir sobre a importância da sistematização para a melhoria da qualidade da assistência de enfermagem (ABRÃ $\left.{ }^{1}, 1993\right)$. No entanto, a falta de indicadores de alteração das necessidades básicas, dificultaram a identificação dos problemas de enfermagem ou elaboração de diagnósticos de enfermagem, segundo este referencial.

O conhecimento da classificação dos diagnósticos de enfermagem propostos pela NANDA e de sua aplicação prática, despertou o interesse dessa autora em aplicá-los nas consultas de enfermagem ${ }^{* * * * * *} \mathrm{em}$ aleitamento materno, de modo a complementar a assistência anteriormente realizada. A oportunidade surgiu no período que cursou a disciplina "Estudos Avançados da Assistência de Enfermagem", no Curso de Doutorado em Enfermagem do Departamento de Enfermagem da Universidade Federal de São Paulo, quando desenvolveu o presente trabalho sob a orientação e com a colaboração das outras autoras.

\section{OBJETIVOS}

Propusemo-nos como objetivos:

- a reformulação de um instrumento de coleta de dados, com base na classificação dos diagnósticos de enfermagem, proposta na taxonomia I-revisada da NANDA;

- a identificação de diagnósticos de enfermagem mais freqüentes, relacionados ao aleitamento materno, a partir da aplicação do instrumento reformulado.

\section{METODOLOGIA}

\section{Local de Estudo}

O presente trabalho foi desenvolvido no ambulatório da Maternidade Amparo Maternal que, atualmente, mantém convênio com a Universidade Federal de São Paulo.

\section{Amostra}

A amostra constitui-se de 12 puérperas que tiveram parto cesárea e que retornaram ao ambulatório do referido Hospital para a primeira consulta pós-alta hospitalar.

\footnotetext{
****** Consideramos diagnóstico e problema de enfermagem como sinônimos, conforme publicações oficiais do International Council of Nurses, 1993

***** Para as autoras a consulta de enfermagem é uma atividade que quando executada de forma sistematizada, tem correspondência com o processo de enfermagem
} 


\section{Coleta de Dados}

A coleta de dados foi feita durante a consulta a puérpera, agendada previamente entre o $7^{\circ}$ e $15^{\circ}$ dia pósparto. Para esta coleta, utilizou-se o instrumento apresentado a seguir.

\section{Instrumento utilizado}

Com o propósito de incorporar alguns avanços que a Taxonomia da NANDA propõe em termos de classificação de diagnóstico de enfermagem, o instrumento utilizado anteriormente nas consultas de enfermagem (Anexo 1), foi reformulado adaptando-o à nova estrutura. A reformulação do instrumento foi feita com base na literatura sobre diagnóstico de enfermagem e aleitamento materno e na experiência anterior das pesquisadoras em sistematização da assistência de enfermagem. Assim, foram selecionados, dentre os Padrões de Resposta Humana, aqueles diagnósticos que considerou-se, terem relação com os problemas da puérpera em fase de amamentação. Analisando-se as definições de cada diagnóstico, as características definidoras e os fatores relacionados, foram selecionados inicialmente 30 diagnósticos de enfermagem. $\mathrm{O}$ instrumento foi aplicado em 5 puérperas e os resultados mostraram a necessidade de incluir alguns itens, excluir outros que não tinham uma relação direta com a amamentação e ainda, reformular a seqüência de modo a adequá-las às etapas da realização da consulta de enfermagem. Desta forma, no instrumento definitivo foram incluídos os itens correspondentes a 25 diagnósticos.

$\mathrm{O}$ instrumento definitivo foi dividido em duas partes, a primeira contendo os itens para a coleta dos dados (Anexo 2), a segunda contendo um guia da pesquisadora, com os 25 diagnósticos de enfermagem (Anexo 3), relacionados segundo cada padrão de resposta humana, os quais apresentamos a seguir:

Trocar: potencial para infecção, hipertemia, integridade da pele prejudicada, potencial para prejuízo da integridade da pele;

Relacionar: interação social prejudicada, alteração no desempenho de papel, paternidade ou maternidade alterada, potencial para paternidade ou maternidade alterada, alteração no processo familiar, conflito no desempenho de papéis dos pais, alteração nos padrões de sexualidade;

Escolher: recusa, conflito de decisão, comportamento para elevar nível de saúde;

Mover: mobilidade física prejudicada, distúrbio no padrão do sono, amamentação ineficaz, fadiga;

Perceber: distúrbio na imagem corporal, baixa autoestima situacional, distúrbio da auto-estima;

Conhecer: déficit de conhecimento;

Sentir: dor, ansiedade, medo.

\section{APRESENTAÇÃO E ANÁLISE DOS RESULTADOS}

Após ter realizado as 12 consultas de enfermagem e concluída a fase de coleta de dados, foi iniciado o trabalho de análise das informações coletadas. O processo utilizado para análise e síntese dos dados até chegar aos diagnósticos teve como base as características definidoras e os fatores relacionados, determinados pela classificação da NANDA, além do conhecimento e experiência das autoras em sistematização da assistência de enfermagem e especificamente em aleitamento materno.

É importante ressaltar que no momento da consulta não foi possível fazer o registro dos diagnósticos nos termos propostos pela NANDA, uma vez que a situação demandava rapidez no atendimento. A mãe recebeu a orientação e assistência necessária no momento da consulta.

Apesar de não se ter estabelecido como objetivo do estudo a caracterização da clientela, julgou-se importante apresentar alguns dados relativos a esse aspecto, por acreditar que eles possam estar relacionados aos diagnósticos identificados e constituir-se objeto de estudos posteriores.

A amostra pesquisada constituiu-se, na maioria, de mulheres entre 15 a 30 anos de idade (91\%), com escolaridade entre $1^{\mathrm{a}}$ e $8^{\mathrm{a}}$ série $(91,6 \%)$ e primíparas $(84 \%)$.

Quanto aos diagnósticos de enfermagem, constatou-se que dos 25 relacionados no instrumento, apenas 13 foram identificados em nossa amostra, os quais estão relacionados na Tabela 1, apresentada a seguir:

Tabela 1 - Diagnósticos de enfermagem relacionados à amamentação identificados no atendimento de 12 puérperas na M.A.M. - SP - 1995

\begin{tabular}{lcc}
\hline DIAGNÓSTICOS DE ENFERMAGEM & $\mathrm{N}^{\circ}$ & $\%$ \\
\hline Déficit de conhecimento & 12 & 100,0 \\
Distúrbio no padrão do sono & 09 & 75,0 \\
Alteração no padrão de sexualidade & 09 & 75,0 \\
Amamentação ine ficaz & 08 & 66,6 \\
Mobilidade fisica prejudicada & 08 & 66,6 \\
Fadiga & 07 & 58,3 \\
Ansiedade & 04 & 33,3 \\
Medo & 02 & 16,6 \\
Potencial para prejuizo da integridade da & 02 & 16,6 \\
pele & & \\
Potencial para infecção & 02 & 16,6 \\
Alteração no processo familiar & 02 & 16,6 \\
Potencial para maternidade alterada & 01 & 8,3 \\
Conflito no desempenho de papéis dos pais & 01 & 8,3 \\
\hline
\end{tabular}


Analisando a Tabela 1, pode-se observar que: - o diagnóstico "déficit de conhecimento" foi identificado em 12 mulheres $(100,0 \%)$, sendo que todas referiram desconhecer ou ter conhecimento incorreto sobre aspectos relacionados à prática do aleitamento materno. Relacionaremos a seguir, os aspectos identificados como déficit de conhecimento: não conhecer as vantagens do aleitamento materno $(75,0 \%)$; não conhecer a estrutura interna da mama e como ocorre a produção do leite $(100,0 \%)$; não conhecer os cuidados com as mamas durante a gestação (100,0\%); não conhecer a técnica de amamentação (91,6\%); não conhecer os procedimentos com a mama na vigência de ingurgitamento mamário $(100,0 \%)$ e traumas mamilares $(75,0 \%)$, e não conhecer os procedimentos com as mamas na vigência da mastite $(100,0 \%)$;

- o diagnóstico "distúrbio no padrão do sono" foi encontrado em 9 puérperas $(75,0 \%)$ e todas elas referiram não estar conseguindo dormir bem a noite porque o recémnascido chorava muito e queria ficar o tempo todo mamando;

- "alteração no padrão de sexualidade" foi manifestado também por 9 mulheres $(75,0 \%)$ sendo que algumas referiram preocupação em iniciar a atividade sexual, por desconhecer outros métodos contraceptivos que poderiam estar utilizando, visto que foram informadas de que não poderiam fazer uso da pílula convencional;

- o diagnóstico "amamentação ineficaz" foi identificado em 8 puérperas $(66,6 \%)$. Algumas faziam uso de chá nos intervalos das mamadas e adotavam o esquema de horário rígido para amamentar, outras já haviam inserido mamadeiras de leite para complementar a alimentação da criança, e uma mãe referiu a utilização do calor úmido para o tratamento do ingurgitamento mamário;

- sete puérperas $(58,3 \%)$ apresentaram o diagnóstico de "fadiga" referindo-se a impossibilidade de descanso noturno por causa da criança e diurno devido aos afazeres domésticos e cuidados com outros filhos;

- quatro mulheres $(33,3 \%)$ apresentaram o diagnóstico "ansiedade" referindo-se a uma sensação ruim, porém sem conseguir definir a causa aparente. Apenas uma puérpera referiu estar ansiosa por causa da proibição do uso do cigarro pelo marido;

- o diagnóstico "medo" foi encontrado em 2 mulheres $(16,6 \%)$ que referiram-no em relação a hipogalactia e ao fato do leite ser "fraco" para a criança;

- "potencial para prejuízo da integridade da pele" ocorreu em apenas duas mulheres (16,5\%), que apresentaram os mamilos hiperemiados devido à prática de higienização dos mesmos antes do ato de amamentar;

- o diagnóstico "potencial para infecção" foi encontrado em 2 puérperas $(16,6 \%)$ que referiam ingurgitamento mamário e apresentaram mamilos hiperemiados;
- "interação social prejudicada" foi identificado, também em duas mulheres $(16,6 \%)$, por referirem desânimo, tristeza e/ou irritação no convívio com outras pessoas que não aquelas mais íntimas;

- "alteração no processo familiar" foi percebida em duas mulheres $(16,6 \%)$ que referiram problemas de relacionamento com o companheiro;

- o diagnóstico "potencial para maternidade alterada" foi encontrado em apenas uma puérpera $(8,3 \%)$ que demonstrou inabilidade e desinteresse para cuidar da criança;

- da mesma forma, o diagnóstico "conflito no desempenho de papéis dos pais" foi identificado em apenas uma puérpera $(8,3 \%)$, porque a mesma mostrou-se relutante na execução dos cuidados com o recém-nascido.

Alguns destes diagnósticos encontrados coincidem, em parte, com estudo realizado por FOLKERMAGLAYA $^{6}$ (1994), que em trabalho semelhante identificou basicamente 4 diagnósticos em aleitamento materno: déficit de conhecimento, dor e desconforto, alto risco de infecção e amamentação ineficaz.

Três destes diagnósticos, coincidiram com os diagnósticos por nós encontrados (amamentação ineficaz, déficit de conhecimento e alto risco de infecção). O diagnóstico de dor e desconforto, não foi encontrado nesta amostra porém, a nossa experiência e o trabalho realizado por ABRÃO ${ }^{1}$ (1993) mostrou que este diagnóstico é muito freqüente na presença de traumas mamilares e ingurgitamento mamário, patologias encontradas neste trabalho, respectivamente em $83,3 \%$ e $70,0 \%$ das mulheres. Por outro lado, temos a considerar, que a população de mulheres americanas tem características diferentes, condições sócio-econômicas e necessidades bem diversas das mulheres por nós pesquisadas e portanto, alguns dos diagnósticos encontrados aqui, poderão não ser encontrados lá, necessitando de estudos de validação destes diagnósticos em mulheres brasileiras.

FOLKER-MAGLAYA $^{6}$ (1994), também faz referência às intervenções de enfermagem realizadas para cada diagnóstico encontrado. Estas orientações são aquelas propostas por estudiosos em aleitamento materno, e coincidem, com as orientações por nós realizadas, quando do momento da consulta. Elas não foram relacionadas no presente trabalho por não constituírem objeto de análise, no entanto, reconhecemos a importância de estudos que tratem da relação entre os diagnósticos, intervenções de enfermagem e resultados alcançados.

Com relação aos demais diagnósticos encontrados em uma ou duas mulheres, recomenda-se que o trabalho seja replicado em outra população e com uma amostra maior para verificar se as freqüências de aparecimento se mantém pequenas ou não e, a partir dos resultados, chegar a novas conclusões. 


\section{CONCLUSÕES E CONSIDERAÇÕES FINAIS}

Diante do que foi exposto, pode-se concluir que os diagnósticos encontrados em percentual acima de $50 \%$ foram: déficit de conhecimento (100\%), distúrbio no padrão do sono (75\%); alteração no padrão de sexualidade (75\%), amamentação ineficaz $(66,6 \%)$ e mobilidade física prejudicada $(66,6 \%)$.

Embora este trabalho represente um estudo preliminar no levantamento dos possíveis diagnósticos de enfermagem identificados em mulheres que amamentam, os diagnósticos mais freqüentes, devem estar contemplados no instrumento de coleta de dados para consulta de enfermagem em aleitamento materno, pois confirmam a sua necessidade.

Os demais diagnósticos devem continuar fazendo parte do instrumento de consulta de enfermagem, a fim de que seja mantida a abrangência necessária à assistência à puérpera.

Finalmente, este trabalho permitiu-nos entender que o diagnóstico não pode ser uma fase isolada de todo o processo assistencial de enfermagem; deve ser utilizado com o objetivo de direcionar a ação de enfermagem para uma resolução ou intervenção. A incorporação dos diagnósticos de enfermagem propostos pela NANDA, permitiu confirmar a concepção que as autoras já possuíam que a assistência em aleitamento materno não pode ser voltada somente para os aspectos biológicos, mas também, para os sociais e psicológicos. Possibilitou ainda, desenvolver uma atenção de enfermagem, não somente voltada às mães, mas ao trinômio mãe-pai-filho, inseridos num contexto familiar.

\section{UTILIZATION OF NURSING DIAGNOSIS ACCORDING TO NANDA'S CLASSIFICATION IN ORDER TO SYSTEMATIZE NURSING CARE IN BREASTFEEDING}

The present study aimed at describing the reformulated instrument used in the puerperal woman nursing consultation based on the identified diagnoses classification according to the Taxonomy-I reviewed by NANDA, and the identification of the most frequent nursing diagnoses concerning maternal breastfeeding, based on the reformulated instrument. The diagnoses found as being over 50\% were: knowledge deficit (100\%); sleep pattern disturbance (75\%), altered sexuality patterns (75\%), ineffective breastfeeding $(66,6 \%)$ and impaired physical mobility $(66,6 \%)$.

\section{UTILIZACIÓN DEL DIAGNÓSTICO DE ENFERMERÍA, SEGÚN CLASIFICACIÓN DE LA NANDA, PARA SISTEMATIZACIÓN DE LA ASISTENCIA DE ENFERMERÍA EN LA LACTANCIA MATERNA}

En este estudio se describe el proceso de reformulación de un guía de evaluación de puérperas, utilizado en la consulta de enfermeria, teniendo como referencia la clasificación de los diagnósticos de enfermería de la Taxonomía I-revisada por la Asociación Norteamericana de Enfermería (NANDA). También son determinados los diagnósticos de enfermería relacionados con la lactancia materna, que fueron calificados con porcentajes superiores a 50\%, a partir de la aplicación de la guía reformulada. Ellos son: déficit de conocimiento (100,0\%), cambios en el patrón del sueño (75,0\%), cambios en el patrón de sexualidad $(75,0 \%)$, lactancia ineficaz $(66,6 \%)$ y movilidad física disminuida.

TÉRMINOS CLAVES: diagnóstico de enfermería, lactancia materna, salud de la mujer

\section{REFERÊNCIAS BIBLIOGRÁFICAS}

01. ABRÃO, A.C.F.V. Aleitamento materno: efeito de um programa educativo para o auto cuidado com as mamas na incidência de ingurgitamento mamário e traumas mamilares. São Paulo, 1993. 110p. Dissertação (Mestrado) - Departamento de Enfermagem, Escola Paulista de Medicina.

02. CAETANO, L.C. Aleitamento materno: fatores que contribuem para sua prática. São Paulo, 1992. 168p. Dissertação (Mestrado) - Departamento de Enfermagem, Escola Paulista de Medicina.
03. CRUZ, D.A.L.M. A introdução do diagnóstico de enfermagem no ensino: sua influência no processo de informações por alunos de graduação. São Paulo, 1995. 149p. Tese (Doutorado). Escola de Enfermagem, Universidade de São Paulo.

04. CRUZ, D.A.L.M. Diagnóstico de enfermagem: aspectos históricos e definição. Rev.Paul.Enfermagem, v.13, n.1/3, p.3-7, 1994.

05. FARIAS, J.N.; NÓBREGA, M.M.L.; PÉREZ, V.L.A.B.; COLER, M.S. Diagnóstico de enfermagem - uma abordagem conceitual e prática. João Pessoa : Santa Marta, 1990. 
06. FOLKER-MAGLAYA, C. Facilitating breastfeeding. In: GULANICH, M.; GRADISHAR, D.; PUZAS, M.K. Obstetric and gynecology nursing plans of care for specialty practice. USA: Delmar Publishers, 1994. p.334-354.

07. GIUGLIANI, E.R.J. Amamentação: como e porque promover. J.Pediatr., v.70, n.3, p.138-147, 1994.

08. HORTA, V.A. Processo de enfermagem. 8 ed. São Paulo: EPU, 1979.

09. IYER, P.; TAPTICH, B.; BERNOCCHI-LOSEY, D. Processo e diagnóstico de enfermagem. Trad. Regina Machado Garcez. Porto Alegre:Artes Médicas, 1993.
10. LETHBRIGE, D.J.; McCLURG, V.; HENRIKSON, M.; WALL, G. Validation of the nursing diagnosis of ineffective brestfeeding. J. Obstet., Ginecol. Neonatal Nurs., v.22, n.1, p.57-63, 1993.

11. MANFREDI, M. Lactancia materna en el proceso de educación en enfermeria/Apresentado no Seminário sobre Lactancia materna, Georgetown, 1989.

12. ORGANIZAÇÃO MUNDIAL DA SAÚDE/ FUNDO DAS NAÇÕES UNIDAS PARA A INFÂNCIA. Protección, promoción y apoyo de la lactancia natural. Genebra, 1989. 


\section{CONSULTA DE ENFERMAGEM EM ALEITAMENTO MATERNO}

(Modelo Wanda Horta)

\section{HISTÓRICO}

1.1. Dados de identificação

- Nome

- Idade

- Estado civil

- Profissão

- Escolaridade

- Endereço completo

- Nome e profissão do cônjugue

- Nome do obstetra

- Telefone para contato

1.2. Antecedentes Obstétricos gesta _ Para Filhos vivos

1.3. Percepções e expectativas da mulher quanto à amamentação

- conhecimento sobre as vantagens do aleitamento materno, anatomia da mama, fisiologia da lactação, técnicas de amamentação, cuidados específicos com a mama lactante normal e mamilos, cuidados com ingurgitamento mamário e traumas mamilares.

experiência anterior em amamentação.

- preparo das mamas e mamilos em gestações anteriores.

- intercorrências anteriores.

1.4. Informações sobre a gestação atual
- D.U.M.
D.P.P
- Preparo das mamas e mamilos
- Intercorrências

1.5. Informações sobre o parto atual

- Data

- Tipo

- Anestesia

- Idade gestacional ao nascer

- Sexo do RN

- Peso do RN

- Intercorrências

- Cuidados realizados com as mamas e mamilos durante a internação

- RN tipo de aleitamento, esquema de horário, complementos

2. EXAME FÍSICO DAS MAMAS

- forma - direito e esquerdo

- tipo de mamilo

- região mamilo-areolar

- presença de colostro

- apojadura

- consistência das mamas

- condições de histórico

3. DADOS CLÍNICOS DE INTERESSE

- medicação em uso

- outras informações

- impressões do entrevistador

4. LEVANTAMENTO DE PROBLEMAS/NECESSIDADES BÁSICAS AFETADAS

\section{PRESCRIÇÃO DE ENFERMAGEM E EVOLUÇÃO}


ANEXO 2

CONSULTA DE ENFERMAGEM EM ALEITAMENTO MATERNO

(NANDA)

\section{HISTÓRICO}

- Dados de identificação

Nome idade

estado civil profissão escolaridade endereço completo nome e profissão do cônjuge

- Antecedentes obstétricos

gesta para filhos vivos

- Gestações anteriores experiência em amamentação preparo das mamas intercorrências $\begin{array}{ll}\operatorname{sim}(\text { ) } & \text { não ( ) } \\ \operatorname{sim}(\text { ) } & \text { não ( ) }\end{array}$

- Informações sobre a gestação atual DUM $\operatorname{sim}($ ) não ( )

duração

tipo quais

Início $\operatorname{sim}($ )

Como?

tercorrências

Medicações em uso

- Informações sobre o parto atual

Data $\longrightarrow$ tipo anestesia

Idade gestacional ao nascer

Peso do RN

Intercorrências

- Informações sobre o conhecimento da mulher quanto a:

vantagens da amamentação $\operatorname{sim}($ ) não ( )

anatomia da mama $\operatorname{sim}($ ) não ( )

fisiologia da lactação $\operatorname{sim}($ ) não ( )

procedimento com a mama durante a gestação $\quad \operatorname{sim}($ ) não ( )

Quais

procedimentos com a mama lactante normal $\operatorname{sim}($ ) não ( )

Quais

procedimentos com a mama na presença de traumas mamilares $\quad \operatorname{sim}()$ não ( )

Quais

procedimentos com a mama na presença de ingurgitamento mamário $\operatorname{sim}($ ) não ( )

Quais

procedimentos com a mama na presença de mastite $\operatorname{sim}(\quad)$ não ( )

Quais

- DIAGNÓSTICO 8.11

3.1.1. Relacionamento com outras pessoas bom ( ) ruim ( ) regular ( )

3.2.1./3.2.1.1.2 - Como você está cuidando do seu filho/gostou do sexo da criança

3.3.1.1.1. Relacionamento com a criança: cuida direito ( ) total ( ) olha ( ) conversa ( )

3.2.2./3.2.3.1. Como está seu relacionamento com o marido e filhos

3.2.1.2.1./3.3. relacionamento sexual

5.2.1.1./5.3.1.1. está amamentando $\operatorname{sim}($ ) não ( ) porque o aleitamento provocou mudanças na sua vida $\operatorname{sim}()$ não () gosta de amamentar $\operatorname{sim}($ ) não ( ) pretende continuar sim ( ) não ( )

5.4. você tem cuidado de si própria $\operatorname{sim}($ ) não ( )

6.1.1.1. movimentação física

6.1.1.2.1. sente-se cansada, desanimada $\operatorname{sim}($ ) não ( ) 
Rev. latino-am. enfermagem - Ribeirão Preto - v. 5 - n. 2 - p. $49-59$ - abril 1997

6.2.1. como está o seu sono dorme à noite toda ( ) acorda várias vezes ( )

dorme de dia ( )

6.5.1.2. Cuidados realizados com as mamas durante a internação e no domicílio:

6.5.1.2. Tipo de aleitamento exclusivo ( ) misto ( ) artificial ( )

6.5.1.2. Esquema de horário de amamentação rígido ( ) livre demanda ( )

6.5.1.2. Uso de complementos $\operatorname{sim}($ ) não ( )

6.5.1.2. Medicações em uso $\operatorname{sim}()$ não ( ) Quais

7.1.1. Como você se vêm amamentando

7.1.2. Auto-estima

7.1.2.2. Você se sente capaz para lidar com essa situação nova

9.1.1. Você sente dor ao amamentar $\operatorname{sim}($ ) não ( )

9.3.1. Neste período de amamentação você se sente preocupada ( ) ansiosa ( ) tensa ( )

9.3.2. Você sente medo ao amamentar $\operatorname{sim}()^{\prime}$ não ( ) Outras

queixas

2. EXAMES FÍSICO DAS MAMAS

- Tipo de mamilo

direito

protruso ( )

semi-protrusor ( )

malformado ( )

D. 1.6.2.1.2.2.

direito

- região mamilo-areolar

D. 1.6.2.1.2.2.

pigmentação normal ( )

- colostro

- apojadura

despigmentada ( )

presente ( )

presente ( )

- consistência das mamas

direito

normal ( )

D. 1.2.1.1./1.6.2.1.2.2.

- condições dos mamilos

ingurgitada ( )

direito

normal ( )

D. 1.6.2.1.2.2.

hiperemiado ( )

D. 1.6.2.1.2.1./1.2.1.1.

traumatizado ( )

\section{esquerdo}

protruso ( )

semi-protruso ( )

malformado ( )

esquerdo

pigmentação normal ( )

despigmentada ( )

ausente ( )

ausente ( )

esquerdo

normal ( )

ingurgitada ( )

esquerdo

normal ( )

hiperemiado ( )

traumatizado ( ) 


\section{ANEXO 3}

\section{GUIA DA PESQUISADORA PARA A IDENTIFICAÇÃO DOS DIAGNÓSTICOS DE ENFERMAGEM}

\subsubsection{Potencial para infecção $\quad$ Presente ( ) Ausente ( ) Não identificados ( )} caracterizado por:

relacionado a:

1.6.2.1.2.1. integridade da pele prejudicada Presente ( ) Ausente ( ) Não identificados ( ) caracterizado por:

relacionado a:

1.6.2.1.2.2. potencial para prejuízo na integridade da pele Presente ( ) Ausente ( ) Não identificados ( ) caracterizado por:

relacionado a:

\subsubsection{Hipertemia Presente ( ) Ausente ( ) Não identificados ( )}

caracterizado por:

relacionado a:

\section{RELACIONAR (3)}

3.1.1. Interação social prejudicada $\quad$ Presente ( ) Ausente ( ) Não identificados ( ) caracterizado por:

relacionado a:

3.2.1. Alteração no desempenho de papel Presente ( ) Ausente ( ) Não identificados ( ) caracterizado por:

relacionado a:

3.2.1.1.1. Maternidade alterada Presente ( ) Ausente ( ) Não identificados ( )

caracterizado por:

relacionado a:

3.2.1.1.2. Potencial para maternidade alterada Presente ( ) Ausente ( ) Não identificados ( ) caracterizado por:

relacionado a:

\subsubsection{Alteração no processo familiar $\quad$ Presente ( ) Ausente ( ) Não identificados ( )}

caracterizado por:

relacionado a:

3.2.3.1. Conflito no desempenho de papéis dos pais Presente ( ) Ausente ( ) Não identificados ( ) caracterizado por:

relacionado a:

3.3. Alteração nos padrões de sexualidade Presente ( ) Ausente ( ) Não identificados ( ) caracterizado por:

relacionado a:

\section{ESCOLHER (5)}

5.2.1.1. Recusa Presente ( ) Ausente ( ) Não identificados ( )

caracterizado por:

relacionado a:
5.3.1.1. Conflito de decisão
Presente ( )
Ausente ( )
Não identificados ( )

caracterizado por:

relacionado a: 
5.4. Comportamento para elevar níveis de saúde $\quad$ Presente ( ) Ausente ( ) Não identificados ( ) caracterizado por:

relacionado a:

\section{MOVER (6)}

6.2.1. Distúrbio no padrão do sono Presente ( ) Ausente ( ) Não identificados ( ) caracterizado por:

relacionado a:

6.1.1.1. Mobilidade física prejudicada Presente ( ) Ausente ( ) Não identificados ( ) caracterizado por:

relacionado a:

6.1.1.2.1 Fadiga Presente ( ) Ausente ( ) Não identificados ( )

caracterizado por:

relacionado a:

6.5.1.2. Amamentação ineficaz Presente ( ) Ausente ( ) Não identificados ( )

caracterizado por:

relacionado a:

\section{PERCEBER (7)}

7.1.1. Distúrbio da imagem corporal Presente ( ) Ausente ( ) Não identificados ( ) caracterizado por:

relacionado a:

7.1.2. Distúrbio da auto-estima Presente ( ) Ausente ( ) Não identificados ( ) caracterizado por:

relacionado a:

7.1.2.2. Baixa auto-estima situacional Presente ( ) Ausente ( ) Não identificados ( )

caracterizado por:

relacionado a:

\section{CONHECER (8)}

8.1.1. Déficit de conhecimento Presente ( ) Ausente ( ) Não identificados ( ) caracterizado por: relacionado a:

\section{SENTIR (9)}

9.1.1. Dor Presente ( ) Ausente ( ) Não identificados ( )

caracterizado por:

relacionado a:

9.3.1. Ansiedade caracterizado por: relacionado a:

9.3.2. Medo Presente ( ) Ausente ( ) Não identificados ( ) caracterizado por:

relacionado a: 